\author{
DGB-Rechtssekretär gefeuert
}

Am 7. 8. 1974 kündigte der DGB-Bundesvorstand dem Rechtssekretär des DGB Mannheim, Stephan Baier, zum 30.9.7 $\neq$ bei sofortiger Suspendierung von seiner Tätigkeit. Die Kündigungsbegründung: "Dir sind ain 17.7.197ł im Gewerkschaftshaus in Stuttgart durch den Leiter der Abteilung Personal der DGB-Bundesvorstandsverwaltung, Kollegen Arnold Wechsel, in Bezug auf Deine gewerkschaftliche Zuverlässigkeit bzw. Dein Loyalitätsverhältnis zum DGB konkrete Fragen gesteilt worden, deren Beantwortung an Ort und Stelle Du in der Mehrzahl abgelehnt hast bei gleichzeitiger Feststellung, daß Du die Fragen schriftlich beantworten würdest. Da die Beantwortung auch trotz unserer schriftichen Aufforderung vom 31.7.1974 bis heute nicht erfolgt ist, sehen wir u. a. auch im Hinblick auf $\$ 2$ Abs. I $c$ der Satzung des DGB keine Möglichkeit einer weiteren vertrauensvollen Zusammenarbeit. "

Diese politische Disziplinierung in Folge der Abgrenzungsbeschlüsse des DGB hat folgende Vorgeschichte: Am 1. Mai 1974 sagte Gerd Muhr, Mitylied des DGB-Bundesvorstandes, auf der Kundgebung des DGB in Mannheim: "Niemals sollten wir vergessen, daß ein 1933 nicht denkbar gewesen wäre ohne die unheilige Allianz zwischen den rechten und den linken Extremen in diesem Lande, deren gemeinsames Ziel die Beseitigung der Demokratie war. * Gegen diese geschichtsklitternde Ansicht protestierte Rechtssekretär Baier laut und keineswegs allein. Dies Verhalten durfte nicht ungeahndet bleiben, vor allem, nachdem verbreitet wurde, Baier habe auf der Kulturveranstaltung des DGB am x. Mai gefordert, das Strebellied zu singen. Dieses Lied setzt sich kritisch mit der Rolle der IG Metall bei der Schließung der Mannheimer Strebelwerke (2000 Beschäftigte) auseinander. Auch sei Baier verantwortlicher Redakteur der Zeitung des Südwestdeutschen Referendarverbandes "Rote Robe". Mit diesen Vorwürfen wurde Baier in einer Kreisvorstandssitzung am 6. Mai konfrontiert, bei der ohne sein Wissen eine Tonbandaufnahme gemacht wurde, deren Herausgabe bis heute verweigert wird. Der DGB setzte unter dem Vorsitz des DGB-Landesvorsitzenden Erlewein eine Kommission ein, um die Anschuldigungen zu überprüfen. Die Vermutung, Baier sei "Chaot, Kommunist oder Anarchist ", wurde im DGB-Haus reichlich gehandelt. Kollege Baier reagierte keineswegs defensiv: In der Sitzung der oben genannten Kommission erklärte er seinen Protest gegen die Rede Muhrs vor allem damit, daß die Auffassung Muhrs ein nachträglicher Schlag ins Gesicht aller Antifaschisten gewesen sei, die im KZ umgebracht wurden. Auf den Vorwurf, er habe das Strebellied gefordert, meinte Baier, er sei $z$ war noch nicht anwesend gewesen, als darüber abgestimmt wurde, er teile aber die Krıtik, daß die IG Metall nicht genug gegen die Stillegung getan habe, nachdem die juristischen Möglichkciten ausgeschöptt waren. Die Kritik der Mitbestimmung im Lied müsse diskutierbar sein, denn 
auch er halte die Mitbestimmung nucht tür geetgnet, die Arbeiterklasse voranzubringen. Schließlich ses er für die "Rote Robe" presserechtlich seit deren Gründung verantwortlich und könne sich nicht vorstellen, daß dieser Zeitschrıft Gewerkschaftsfeindlichkeıt vorgeworfen werden könne. Die Diskussıon über diese Stellungnahme brachte allerles traurige Kuriositäten zutage. So wurde z. B. die Geschichtsauffassung Muhrs von den Anwesenden bestätigt und die dagegen ins Feld geführte Tatsache, daß der ADGB 1933 zu den von den Nazis organisierten Maikundgebungen aufgerufen hat, mit der Bemerkung zurückgewiesen, die Gewerkschaften seien am I. Mai 1933 bereits aufgelöst gewesen. Der DGB-Landesvorsitzende Erlewein kommentierte die Aussage Baiers, er sehe seme Aufgabe darin, die Interessen der Lohnabhängıgen zu vertreten und daher sei seine Tätigkeit gegen die Unternehmer gerichtet, mit der Frage, ob Baier Art. $1_{4}$ Grundgesetz (Eigentumsgarantie) kenne und auf dem Boden der freiheitlich-demokratischen Grundordnung stehe. Uberrascht von dem entschiedenen Auftreten eines DGB-Funktionärs, versammelte sich der Kreiszorstand am 7.6.74 und beschloß vertraulich und eınstımmı: "Die Vorgänge am 1. Mai und das Gespräch mit dem Kollegen Baier haben uns gezeigt, daß der Kollege Baier dringend an seine dienstlichen Pflichten als Gewerkschaftssekretär ermahnt werden muß. Der Kreisvorstand bittet den Landesbezirksvorstand, durch geeignete Mittel festzustellen, ob der Kollege Baier als Gewerkschaftssekretär überhaupt tragbar ist, da der Verdacht besteht, daß seine innere Einstellung zu den Gewerkschaften negativ ist." Eine Veröffentlichung dieses Beschlusses erfolgte nicht. Baier wurde daraufhin am 17.7.1974 zu einem Gespräch mit Vertretern des DGB-Bundesvorstandes, des Landesbezirksvorstandes und des Betriebsrates nach Stuttgart geladen. Drei Fragen standen dort im Mittelpunkt: Erstens, ob Bater Mitglied des KBW sei; zweitens, ob er sich mit den Leıtsätzen zur Arbeıt in den Gewerkschaften des KBW identifiziere und drittens, wie er zu $\$ 2$ Abs. I Ziffer $c$ der Satzung des DGB stehe (Bekenntnis zur freiheitlich-demokratischen Grundordnung). Baier stellte klar, daß zumindest die ersten beiden Fragen unzulässig seien, da sie Gesınnung erforschen würden. Er werde aber dennoch alle Fragen schriftlich beantworten. Damit gaben sich die DGB-Vertreter zufrieden. Eine Frist zur Beantwortung wurde nicht gestellt. Baier fuhr erst mal in den Urlaub, und jetzt passierte es: Obwohl dem DGB bekannt war, daß Baier vom 26. 7.-23.8. in Urlaub war, wurde am 31.7 .74 in einem Schreiben ultimativ gefordert, er solle bis zum 7.8.74 die Fragen schriftlich beantworten. Die prompt folgende Kündigung trägt den Poststempel vom 7.8., d. h. sie wurde zu einem Zeitpunkt verfaßt, zu dem theoretisch eine Stellungnahme noch möglich gewesen wäre. Der DGBBundesvorstand war sich offensichtlich seiner Trickserei bewußt. Kündigungsgrund war nun nicht mehr in erster Linie die angezweifelte Gewerkschaftstreue, sondern die Nichteinhaltung einer Frist.

Kollege Baier erfuhr von dieser Post erst, als er nach seinem Urlaub die Arbeit wieder aufnehmen wollte. Die sofortige Suspendierung bewirkte, daß die laufenden Arbeitsgerichtsprozesse nur ungenügend erledigt wurden und die Rechtsberatung der Kollegen behindert war. Daß so letztlich Kollegen, die betriebliche Kontlikte durchzustehen hatten, die Leidtragenden waren, erschien dem DGB-Bundesvorstand offenbar zweitrangig. Der Betriebsrat des $D G B$ wurde zu dem im Kündigungsschreiben genannten Kündigungsgrund nicht gehört.

Baier, der nach Meinung vieler haupt- und ehrenamtlicher Kollegen der erfolgreichste Rechtssekretär seit Jahren ist, erhob Kündigungsschutzklage und bean- 
tragte eine Einstweilige Verfügung, mit der erreucht werden sollte, dals er mindestens bis zum 31. I 2.74 (dem ordnungsgemäßen Kündigungstermin) seiner Arbeit weiter nachgehen konnte. Dieses entschiedene Auftreten erstaunte manchen Gewerkschaftsfunktionär; zu einer Solidaritätserklärung von jenen, die sonst bei jeder Gelegenheit das Wort Solidarität benutzen, wenn andere sie üben sollen, kam es allerdings nicht. Eingeschworen auf legalistische Ideologien, wollte man erst einmal sehen, wem das Gericht recht gibt.

In dem Güte- und sogleich nachfolgenden Kammertermin ( $\$$ SS Abs. I ArbGG) vor dem Arbeitsgericht Mannheim am 11.9.74 erklärte Baier u.a.: *Ich bedauere, vor einem staatlichen Gericht, in dem auch ein Vertreter der Arbeitgeberverbände Sitz und Stimme hat, innergewerkschaftliche Fragen zu erörtern, deren Behandlung eigentlich in die Gewerkschaft gehört. Ich würde hier freiwillig auf kontroverse Fragen der Gewerkschaftspolitik auch nicht eingehen, werde aber von Euch zu dieser Diskussion gezwungen, da die Kündigung nicht auf Handlungen oder Mängel in meiner Arbeit als Rechtssekretär, sondern ausschließlich auf meine (vermuteten) Ansichten gestützt wird... Mit den Prozessen, die ich für unsere Gewerkschaftsmitglieder bisher geführt habe und die noch nicht abgeschlossen sind, sieht es dagegen nicht rosig aus. Ich bekomme zur Zeit manchmal besorgte, teilweise auch empörte Anrufe von Kollegen, die meinen, es bekomme ihren Arbeitsgerichtsverfahren nicht gut, wenn ihr Prozeßvertreter in der entscheidenden Phase des Prozesses so einfach ausgewechselt wird. Und die Kollegen wissen noch nicht alles: daß mir verwehrt wurde, meinem Kollegen in der Rechtsstelle ordentlich die Akten zu übergeben, das heißt, ihn über den Stand der Verfahren und deren Erfolgsaussichten zu unterrichten. Gegen das Verbot, wenigstens bis zum Ablauf der Kündigungsfrist noch für unsere Gewerkschaftskollegen tätig zu sein, habe ich daher eine Einstweilige Verfügung beantragt. Ich wehre mich dagegen, von Gewerkschaftsbeiträgen für den reinen Müßiggang bezahlt zu werden. Auch wurmt es mich zu wissen, daß mein Kollege in der Rechtsstelle jetzt auch noch meine Arbeit übernehmen muß. Manche meiner Kollegen scheiten mich einen ausgemachten Trottel; ich hätte sofort alles unterschreiben sollen, was mir vorgelegt wird, dann könnte ich unseren Kollegen heute noch nützlich sein. Das hat etwas für sich. Sicher ist es töricht, sich selbstmörderisch in eine Kündigung oder ein Ausschlußverfahren zu stürzen wie die Lemminge in das Meer. Das war auch nicht meine Absicht. Aber wenn ich sehe, wie mir Kollegen aus dem Gewerkschaftshaus mit vorgehaltener Hand, unter vier Augen und dem Siege! der Verschwiegenheit ihre Empörung über Euer Vorgehen versichern, dann bestärkt mich das, Euren Fragen Widerstand entgegenzusetzen. Ihr verlangt von mir ein politisches Glaubensbekenntnis und forscht in meiner Gesinnung. Ich wehre mich aber dagegen, von Euch in den Beichtstuhl oder in die Inquisition gedrängt zu werden. Die Mitgliedschaft in einer Gewerkschaft und die Arbeit für Gewerkschaftsmitglieder darf nicht von politischen Bekenntnissen abhängig gemacht werden. Der Kampf um mehr Lohn und bessere Arbeitsbedingungen verträgt es nicht, daß Gewerkschafstmitglieder nach Bibel oder Manifest abgeklopft werden. Eine solche Gesinnungsschnüffelei erzeugt ein Klima von Mißtrauen unter den Kollegen und öffnet der Denunziation Tür und Tor. Das kann schließlich soweit führen, daß einer ausgeschlossen wird, weil man abgegriffene Leninbände in seinem Bücherschrank gesehen hat. * Baier erklärte daraufhin, daß diese Entwicklung zum sozialdemokratischen Gesinnungsverein führe und äußerte sich zum Schluß zur Aufforderung, eine Aussage zum Bekenntnis des DGB in seiner Satzung zur freiheitlich-demokrati- 
schen Grundordnung zu machen: "Allerdings habe ich oft den Eindruck, daß bei solchen Aufforderungen Freiheit und Demokratıe als Köder ausgelegt werden, um die, die danach verlangen, sicher an die Leine der kapitalistischen Wirtschafts- und Gesellschaftsordnung zu legen. Mit der inflationären Verwendung dieses Begriffs wird die Sehnsucht im Volk nach Freiheit und Demokratie dazu milsbraucht, das Volk auf die Zustimmung zu der gegenwärtigen unmenschlichen Produktionsweise festzunagein.

Manche versuchen, sich diesem Zwang dadurch zu entziehen, daß sie in die Verfassung reingreifen wie in einen Selbstbedienungsladen und einem Eigentumsartikel einen Sozialisierungsartikel entgegenstrecken. Richtiger scheint mir, daß Freiheit, Gleichhent und Brüderlichkeit in unserer Gesellschaft verwirklicht sind, allerdings für die beiden entgegengesetzten Klassen auf ganz unterschiedliche Weise.

So haben alle Arbeiter die Freiheit, ihre Arbeitskraft verkaufen zu müssen, so wie alle Kapitalisten die Freiheit haben, deren Arbeitskraft ausbeuten zu dürfen. So hat jeder Arbeiter wie jeder Kapitalist das gleiche Recht, Springers Bild-Zeitung aufzukaufen. So gibt es eine Brüderlichkeit in der Raffgier, mit der die Aktionäre die Gewinne unter sich verteilen, aber auch die weitaus eindrucksvollere Solidarıtät der Arbeiter, mit der sie im Streik um mehr Lohn kämpfen.

Grundlage und Ursache dieser so verschiedenen Freiheit, Gleichheit und Bruderlichkeit ist das Eigentum an Produktionsmitteln. Daß dieses private Eigencum einer allgemeinen Freiheit und einer umfassenden Demokratie entgegensteht, wird wohl kaum ein Gewerkschaftsmitglied ernstlich bestreiten können. Es wurde auch auf der Veranstaltung der IG Metall in Stuttgart anläßlich der erreichten Mitgliederzahl yon 500000 nachdrücklich bekräftigt. $[\cdots]$

Heute frage ich mich, wie es mir nur gelingen konnte, 400 Kollegen derart zu übertölpeln, daß sie es unterließen, an mich Eure Fragen zu richten, bevor sie mir Vollmache zu ihrer Vertretung vor den Arbeitsgerichten erteilten. Ich werde aber den Gedanken nicht los, daß ihnen solche Fragen nicht gerade auf den Nägeln brannten. Meine Aufgabe war es, ihre Interessen vor Gericht zu vertreten. Sollte ich da versagt oder mich gewerkschaftsfeindlich verhalten haben, so haben die Gewerkschaftsmitglieder ein Recht, mich zur Rechenschaft zu ziehen. Es ist eine Selbstverständlichkeit, daß ich mich jeder für alle Gewerkschaftsmitglieder offenen Diskussion hierüber stellen werde. Meine Erfahrungen sagen mir allerdings, daß solche öffentlichen Diskussionen gegen den heftigen Widerstand der derzeitigen Gewerkschaftsführung durchgesetzt werden müßten.*

Der Rechtsanwalt des DGB-Bundesvorstandes, der aus Essen angereist kam, wiederholte die bereits bekannten Vorwürfe und erstaunte sich im übrigen über das Vorgehen des Gerichts, das sofort zu einer Entscheidung kommen wollte. Das Arbeitsgericht Mannheim gab der Klage Baiers statt und verurteilte den DGB zur Weiterbeschäftigung. Das Gericht vertrat die Auffassung, daß die sechswöchige Kündigungsfrist nicht gewahrt sei, da die Kündigung erst am 26.8. 74 - nach Ablauf des Urlaubs - zugegangen und somit eine Kündigung zum 30.9. unmöglich sei. In formeller Hinsicht beanstandete es die nicht ordnungsyemälße Anhörung des Betriebsrates gem. $\$ 102$ Abs. \& BetrVerfG. Materiell sah das Gericht die Kündigung für nicht sozial gerechtfertigt an. Aus der Nichtenhaltung einer Frist könne nicht auf die Sympathisantenschaft zum KBW geschlossen werden. Die Nichtbeantwortung der vom DGB gestellten 
Fragen vor Urlaubsantritt bewertete das Gericht so: nWeiterhin können sich aber auch daraus keinerlei Schlüsse gegen den Kläger herleiten lassen, daß er die Zeit zwischen dem 17.7.1974 und 26.7.1974 nicht etwa zur Abgabe der von ihm zugesagten Äußerung genutzt habe. Hierfür spricht bereits einmal, daß die Beklagtenvertreter bei der Besprechung in Stuttgart dem Kläger keinerlei Frist gesetzt hatten und er mithin davon ausgehen durfte, daß diese Sache nicht allzu sehr eile. Zum anderen hatte er auch bis zum Urlaubsantritt noch enne solche Menge von Arbeit, daß er schlechterdings alles weniger Wichtige etwas hintanstellen konnte. Dabei ging er nach Ansicht der Kammer zu Recht davon aus, daß auch die Beantwortung der ihm gestellten Fragen zu den etwas weniger wichtigen Aufgaben zähle. Es mußte nänilich nach ihrer Ansicht auch dem Beklagten ungemein viel daran gelegen sein, sowohl seine Mitglieder, als auch die Mitarbeiter des Klägers durch dessen Arbeit zufriedengestellt zu sehen. Das Interesse an diesen Zielen mußte dem objektiven Beobachter bei weitem wichtiger scheinen, als eine mehr oder weniger schnell erfolgte Antwort auf die gestellten Fragen. (ArbG Mannheim I Ca 522/74). Konnte man so noch erstaunt sein, daß ein Arbeitsgericht die Interessenpriorität einer Gewerkschaft klar faßt, kam die Berufung des DGB Bundesvorstandes gegen dieses Urteil kaum überraschend. Begründet wurde sie mit der oppositionellen Haltung Baiers und dessen vermuteter Mitgliedschaft im KBW. Darin heißt es: "Zur Vermeidung von Mißverständnissen sei betont, daß selbstverständlich niemand vom Kläger verlangt, er habe stets hundertprozentig mit den Ansichten iibereinzustimmen, die Mitglieder des DGB-Bundesvorstandes äußern. Es steht dem Kläger frei, Meinungen von Mitgliedern des Bundesvorstandes oder des Landesbezirksvorstandes für falsch zu halten und auch zu kritisieren. Nur muß vom Kläger - wie auch anderen hauptberuflichen Arbeitnehmern des Beklagten - verlangt werden, daß er etwaige Kritik nicht in einer gewerkschaftsschädigenden Form äußert.*

Was gewerkschaftsschädigendes Verhalten ist, bestimmt - wie kann man es anders erwarten - der Angegriffene. Zur Veranschaulichung, daß die Zielsetzung des KBW im Gegensatz zur Berufung des DGB-Programms auf die freiheitlich-demokratische Ordnung steht, verweist die Berufungsschrift auf den Jahresbericht 1973 des Bundesamtes für Verfassungsschutz. Zur Erklärung des Kollegen Baier vor dem Arbeitsgericht Mannheim stellte der DGB lakonisch fest: «Die Erklärung vom 11.9.74 bildet... einen neuen Kündigungsgrund, auf den die Beklagte eine weitere, vorsorglich auszusprechende Kündigung zu stützen beabsichtigt. *

Diese Kündigung wurde am 7. II.74 zum 31. I 2.74 ausgesprochen. Inzwischen regte sich allerdings im DGB Mannheim Widerspruch gegen die Maßnahmen des Bundesvorstandes. Betriebsräte und Vertrauensleute von vier Chemiebetrieben Mannheims forderten eine Funktionärs- und Mitgliederversammlung, zu der Kollege Baier geladen werden sollte, um Gelegenheit zu erhalten, zu den gegen ihn erhobenen Vorwürfen Stellung zu nehmen. In einer Vertrauensleuteversammlung der Fa. Braas und Co. mußten die Vorstandsmitglieder der IG Chemie Verwaltungsstelle Mannheim, die diese Forderung kritisierten, zur Kenntnis nehmen, daß keiner der anwesenden 40-60 Vertrauensleute bereit war, sich von dieser Aufforderung zu distanzieren. Auf der Kreisdelegiertenkonferenz des DGB Mannheim am 28. 10.74 sprach sich bei der Diskussion um die Kündigung die große Mehrleit der Redner für einen Verbleib Baiers in der Rechtsstelle aus, ohne daß es allerdings gelang, eine entsprechende Entschließung zur Abstimmung zu stellen. Der Kreisvorstand des DGB faßste darauthin 
den Beschluß, den DGB Bundesvorstand aufzufordern, Baier bis zum Abshluts der Kündigungsschutzprozesse weiterzubeschäftigen. Ein weitergehender Antrag, sich unabhängig von der Entscheidung des Arbeitsgerichts für den Verbleib Baiers einzusetzen, wurde mit 6:6 Stimmen abgelehnt. Die OTV erteilte ihrem Mitglied Baier Rechtsschutz gegen den DGB. Kollegen der IG Chemie starteten im Dezember eine weitere Unterschriftenaktion, in der es u. a. heißt: -Viele Arbeitsgerichtsprozesse stehen zur Zeit an. Gerade jetzt, wo die Kapitalisten die Krise ihrer Wirtschaft auf dem Rücken der Kollegen austragen, haben die Gerverkschaftsmitglieder einen guten Vertreter vor dem Arbeitsgencht nötiger als je zuvor. Kollege Baier hat in dieser Hinsicht gute Arbeit geleistet. Seine Arbeit wurde nie beanstandet, sondern einheilig gelobt. Gleichgültig, welche politischen Auffassungen Kollege Baier hat oder in welcher politischen Organisation er Mitylied ist, trete ich dafür ein, daß er weiter die Interessen der Gewerkschaftsmitglieder vor Arbeitsgerichten vertritt und unsere Interessen gegen die Unternehmer verteidigt.* Um die gleiche Zeit erstritt Baier einen aufsehenerregenden Vergleich für 6 Arbeiterinnen der Firma Vereinigte Offsetdrucker, die sich im Rahmen einer Zusammenlegung $z$ weier Betriebsteile gegen den $18 \mathrm{~km}$ längeren Arbeitsweg gewehrt hatten, enne Abfindung nach III BetrVerfG von zusammen 82000 DM. Den DGB Bundesvorstand kümmerte solch erfolgreiche Interessenvertretung, die Proteste von Kollegen und die Aufforderung des DGB Kressvorstandes nicht, galt es doch, diesen unliebsamen Linken endlich hinauszusäubern. In einer 23 seitigen Begründung der 2. Kündigung, in der neben der Wiederholung aller bisherigen Vorwürfe, wie angekündigt, auch Baiers Stellungnahme vor dem Arbeitsgericht mitverwertet wurde, wird vor allem die ablehnende Haltung Baiers zur Mitbestimmung betont: "Die Frage, ob es in der laufenden Legislaturperiode des Bundestages gelingt, die alte gewerkschaftliche Forderung nach Einführung einer paritätischen Mitbestimmung zu verwirhlichen, steht politisch auf des Messers Schneide. In dieser Situation kann es sich der Beklagte - eine Interessen- und Kampforganisation der Arbeitnehmerschaft - nicht leisten, daß seine eigenen hauptberuflichen Funktionäre für sich das Recht in Anspruch nehmen, die Mitbestimmungsforderung entgegen aller vorliegenden Gewerkschaftsbeschlüsse öffentlich zu kritisieren und abzulehnen. Würde der Beklagte dies hinnehmen, dann würde dies eine empfindliche Schwächung seiner politischen Schlagkraft bedeuten. Es kommt für den Beklagten gerade in der augenblicklich gegebenen politischen Situation darauf an, daß die gesamte Organisation des DGB einheitlich und geschlossen hinter der Forderung nach Einführung der Mitbestimmung steht. «

Während man vorher nur die Form der Kritik bemängelte, war der DGB Bundesvorstand inzwischen inhaltlich geworden. Eine grundsätzliche Kritik an der Mitbestimmung wird nun als gewerkschaftsschadigend diffamiert.

In der mündlichen Begründung der Kündigung vor dem Arbeitsgericht nannte der DGB-Vertreter als weitere Sünde Baiers den Abdruck seiner öffentlichen Erklärung vom ersten Prozeß in der komnunistischen Volkszeitung, sowic den Artikel im express, der eine so intime Detailkenntnis der Vorgänge verrate, daß nur Baier als Verfasser in Frage käme. Während der Gerichtsreporter des express, Heinz Hauser, schmunzelnd und erzürnt diesen Angriff auf die Informationsfreiheit anderer mitschrieb, setzte das Arbeitsgericht durch Beschluß den 2. Kündigungsprozeß bis zur Erledigung der Berufungssache aus. Baier steht seit I. I. 1975 auf der Stralie und hat nun die Zwangsvollstreckung aus dem ersten Arbeitsgerichtsurteil beantragt, um die Weiterbeschäftigung bei 
Meidung einer Geldstrafe zu erzwingen. Auch in diesem Antrag ließ Kollege Baier seine gewerkschaftliche Solidarität walten: $₫ D a$ die finanziellen Mittel des DGB einzig aus Beiträgen von Gewerkschaftsmitgliedern stammen und die Geldstrafe bei Verwirkung dem Staat zufiele, andererseits kein Gewerkschaftsmitglied ein Interesse daran haben kann, mit seinen Beiträgen den Staatshaushalt zu finanzieren, erscheint eine Geldstrafe in Höhe von DM 10,- angebracht. Für eine geringere Summe sich in Bewegung zu setzen, kann keinem Gerichtsvollzieher zugemutet werden."

Baier kandidiert inzwischen auf einer Liste des KBW zum Gemeinderat in Mannheim, was seinen Gewerkschaftsausschluß vorprogrammieren dürfte und wartet auf die Zulassung als Rcchtsanwalt, die er nach Erhalt der 1. Kündigung beantragt hatte und die ihm nun verweigert wird. Zu diesem Vorgang äußerte er sich in einer Presseerklärung vom 30.11.74: Am 25.10.74 erfuhr ich von der Rechtsanwaltskammer Nordbaden, daß sie am 8. I0. vom Justizministerium Baden-Württemberg von meiner Zulassung in Kenntnis gesetzt worden sei; ich sei daraufhin in die Liste der Rechtsanwälte eingetragen worden. Als ich am 27. Ir. im Justizministerium anrief, um zu erfahren, warum ich meine Zulassungsurkunde noch nicht in Händen hätte, teilte mir Regierungsdirektor Storz folgendes mit: Das Zulassungsschreiben sei vom Justizministerium beim Landgericht Heidelberg angehalten worden. Wegen meiner Mitgliedschaft im KBW bestünde der Verdacht, ich würde die freiheitlich-demokratische Grundordnung in strafbarer Weise (!) bekämpfen. Dem Bundesvorstand des DGB reichte für seine Kündigungen anscheinend bereits die Vorstellung, ich könnte Auffassungen für richtig halten, die vom KBW vertreten werden. Beließ es der Bundesvorstand des DGB bei einem Verdacht, so steht für das Justizministerium bereits fest, daß ich Mitglied bin. Es zieht nun in Erwägung, die Mitgliedschaft in einer politischen Organisation - der KBW ist eine Partei - sei unvereinbar mit der Zulassung als Rechtsanwalt ...*

Exemplarisch zeigt das Vorgehen gegen Stefan Baier, wie die Berufsverbote ihre Fortsetzung in den Gewerkschaften und den "freien * Berufen finden.

Ahnlich wie im Offentlichen Dienst werden so auch in den Gewerkschaften die Bedingungen für Sozialisten schlechter, die des Duckmäusertums aber erheblich verbessert.

Heinz Hauser

\section{Zum Tod des Frankfurter Mietrichters Dietmar Kupke}

Der Frankfurter Richter Dr. Dietmar Kupke, der sich nach konfliktreichem Wirken in der Justiz zwischen dem 6. 12. und dem 8.12.1974 das Leben genommen hat, war in vieler Hinsicht eine Ausnahmeerscheinung unter den Richtern dieser Republik.

Er wollte nicht befördert werden und geißeite stattdessen den "Fluch des Beförderungssystems^, das die richterliche Unabhängigkeit zu einer Fiktion mache. In den unruhigsten Zeiten des Frankfurter Häuserkampfes begründete er als einer unter fünf Amtsrichtern in Mietrechtssachen eine Tradition mieterfreundlicher Rechtsprechung, war (im Fall Kettenhofweg 51) öffentlichkeitsfreundlich, wo manch anderer Richter ein handliches, hauseigenes Publikum vorgezogen hätte und gab immer noch keine Ruhe, als er schließlich als Berichter- 